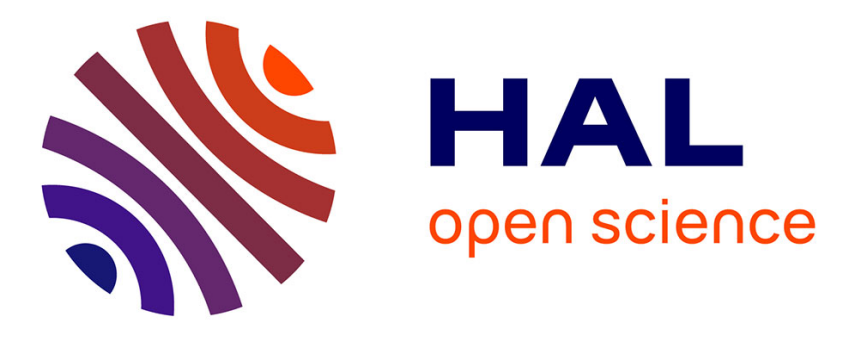

\title{
A theoretical study of the boundary conditions for parabolic equation
}

Rémi Douvenot, Christophe Morlaas, Alexandre Chabory

\section{To cite this version:}

Rémi Douvenot, Christophe Morlaas, Alexandre Chabory. A theoretical study of the boundary conditions for parabolic equation. APWC 2013, IEEE-APS Topical Conference on Antennas and Propagation in Wireless Communications, Sep 2013, France. pp 684 - 687, 10.1109/APWC.2013.6624898 . hal-01073324

\section{HAL Id: hal-01073324 \\ https://hal-enac.archives-ouvertes.fr/hal-01073324}

Submitted on 9 Oct 2014

HAL is a multi-disciplinary open access archive for the deposit and dissemination of scientific research documents, whether they are published or not. The documents may come from teaching and research institutions in France or abroad, or from public or private research centers.
L'archive ouverte pluridisciplinaire HAL, est destinée au dépôt et à la diffusion de documents scientifiques de niveau recherche, publiés ou non, émanant des établissements d'enseignement et de recherche français ou étrangers, des laboratoires publics ou privés. 


\title{
A Theoretical Study of the Boundary Conditions for Parabolic Equation
}

\author{
R. Douvenot, C. Morlaas, and A. Chabory,*
}

\begin{abstract}
For many years, parabolic equation simulations have been performed for $2 \mathrm{D}$ propagation of electromagnetic waves with a great accuracy. However, considering precisely the ground reflection is still an open problem. A classical solution is to consider at each step a constant impedance that depends on the grazing angle calculated by MUSIC or a ray-launching algorithm. For non-constant impedance conditions, this question is not solved. Numerical instabilities may occur when the reflection coefficient is exactly taken into account. This paper theoretically studies this instability. First, the Green function of the vertical operator used for the split-step computation of the propagation is expressed, taking into account the sampling induced by numerical simulations. From this, the observed numerical instability is related to a radiation condition in the upper medium.
\end{abstract}

\section{Introduction}

The parabolic equation algorithm for the simulation of electromagnetic waves propagation in largescale $2 \mathrm{D}$ scenes is an efficient tool [1]. As soon as the backscattered field is neglected, and the propagating power is confined in a cone of revolution [2], the accuracy of the method is well admitted. With the development of the mixed-term Fourier transform [2] and its discrete form [3], the method has also become CPU-efficient. Consequently, it is widely used for propagation in the troposphere [4], $[5]$.

The boundary condition at the ground level is a critical point of the method. Even if it is considered as constant with respect to the vertical spectrum, a numerical divergence problem can occur, sometimes called the "bad alpha" problem, and for which solutions have been proposed [6], [7]. However, considering the impedance at the ground level as constant with the incidence angle is generally an approximation, specially when roughness is introduced in the reflection coefficient (for instance with the Miller-Brown-Vegh (MBV) model [8]).

Efforts have been made to enlarge the theoretical framework of parabolic equation. It has been extended to non-constant impedance conditions [9], and finally to non-constant impedance conditions

${ }^{*}$ The authors are with ENAC, TELECOM/EMA, F31055 Toulouse, France and Toulouse University, F-31400 Toulouse, France.

E-mail: remi.douvenot@recherche.enac.fr in a sampled and bounded domain of computation [10]. It can be implemented with a classical fast-Fourier transform (FFT) method [9]. Lately, a split-step matrix (SSM) transformation algorithm has been proposed [11]. Unfortunately, these algorithms (FFT and SSM) appear to be numerically unstable for some configurations.

In this paper, the expression of the Green function is detailed in section 2 for the sampled and bounded domain of computation. The consequences on what happens beyond the computation domain are studied in section 3.1. Then, a modified permittivity that takes into account the roughness introduced in the reflection coefficient by the MBV model is expressed (section 3.2). Last, in section 3.3, a reflection coefficient inducing numerical instabilities is related to a radiation at infinity in the medium beyond the domain using the modified permittivity. In section 4 , simulation results are presented to illustrate this fact.

\section{The Green function}

Transverse magnetic fields with respect to the $z$ axis are considered, in the time-harmonic domain. A rotational symmetry around the $z$-axis is assumed, and cylindrical coordinates $(r, \phi, z)$ are used, with unit vectors $(\hat{\boldsymbol{r}}, \hat{\boldsymbol{\phi}}, \hat{\boldsymbol{z}})$. The fields are computed in a medium characterised by the physical constants $\left(\varepsilon_{\mathrm{ra}}, \mu_{0}, \sigma_{\mathrm{a}}\right)$ above a ground layer characterised by $\left(\varepsilon_{\mathrm{rg}}, \mu_{0}, \sigma_{\mathrm{g}}\right)$. The computation is performed for altitudes $z \in[0, h]$.

For the computation of the vertical operator, the three-step resolution of the associated StourmLiouville problem [12] is applied. This paper focuses in the first step that consists in calculating the Green function $G_{\lambda}\left(z, z^{\prime}\right)$ of the operator $\mathcal{L}_{z}-\lambda I$, where

$$
\mathcal{L}_{z} \Psi=\left(-\frac{\partial^{2}}{\partial z^{2}}-k^{2}(z)\right) \Psi,
$$

the potential vector being $\Psi$ along $\hat{z}$. The other steps are detailed by Chabory et al. [10]. The Green function $G_{\lambda}\left(z, z^{\prime}\right)$ is defined by

$$
-\frac{\partial}{\partial z^{2}} G_{\lambda}\left(z, z^{\prime}\right)-\left(k^{2}+\lambda\right) G_{\lambda}\left(z, z^{\prime}\right)=\delta\left(z-z^{\prime}\right),
$$

for $\left(z, z^{\prime}\right) \in \mathbb{R}^{2}, z^{\prime}$ being the altitude of the localised source. From equation (2), the Green function is of 
the form

$$
\begin{array}{ll}
G_{\lambda}\left(z, z^{\prime}\right)= & \\
a_{11} e^{-j k_{z \mathrm{a}} z}+a_{12} e^{j k_{z \mathrm{a}} z}, & \text { for } 0<z<z^{\prime}, \\
a_{21} e^{-j k_{z \mathrm{a}}(z-h)}+a_{22} e^{j k_{z \mathrm{a}}(z-h)}, & \text { for } z^{\prime}<z<h,
\end{array}
$$

with $a_{i j}$ constants. $k_{z \mathrm{a}}=\sqrt{k_{\mathrm{a}}^{2}+\lambda}$ is the wavenumber along $z$ into the propagating medium (usually the atmosphere).

At $z=0$, a reflection coefficient $\Gamma$ is introduced to take into account the wave reflected by the ground. At $z=z^{\prime}$, continuity/jump conditions are satisfied. The boundary condition at $z=0$ renders

$$
\begin{gathered}
\lim _{z \rightarrow 0^{+}} \frac{\partial}{\partial z} G_{\lambda}\left(z, z^{\prime}\right)-\lim _{z \rightarrow 0^{-}} \frac{\partial}{\partial z} G_{\lambda}\left(z, z^{\prime}\right)=0, \\
\Rightarrow \quad a_{11}=\Gamma a_{12} \text { with } \Gamma=\frac{k_{z \mathrm{a}}-\epsilon k_{z \mathrm{~g}}}{k_{z \mathrm{a}}+\epsilon k_{z \mathrm{~g}}}
\end{gathered}
$$

where $k_{z \mathrm{~g}}=\sqrt{k_{\mathrm{g}}+\lambda}$ is the the wavenumber along $z$ into the ground medium and $\epsilon=\varepsilon_{\mathrm{a}} / \varepsilon_{\mathrm{g}}$ is the dielectric contrast.

At $z=h$, the domain is bounded by imposing the condition

$$
\frac{\partial G_{\lambda}\left(h, z^{\prime}\right)}{\partial z}-j k_{z \mathrm{a}} \frac{1-\Gamma}{1+\Gamma} G_{\lambda}\left(h, z^{\prime}\right)=0 .
$$

Under this condition, the coefficients $a_{i j}$ are calculated using the jump/continuity conditions. Their expression is

$$
a_{11}=\frac{-1}{4 k_{z \mathrm{a}} \sin \left(k_{z \mathrm{a}} h\right)}\left(\Gamma e^{-j k_{z \mathrm{a}}\left(z^{\prime}-h\right)}+e^{j k_{z \mathrm{a}}\left(z^{\prime}-h\right)}\right),
$$$$
a_{12}=\frac{-1}{4 k_{z \mathrm{a}} \Gamma \sin \left(k_{z \mathrm{a}} h\right)}\left(\Gamma e^{-j k_{z \mathrm{a}}\left(z^{\prime}-h\right)}+e^{j k_{z \mathrm{a}}\left(z^{\prime}-h\right)}\right)
$$$$
a_{21}=\frac{-1}{4 k_{z \mathrm{a}} \sin \left(k_{z \mathrm{a}} h\right)}\left(\Gamma e^{-j k_{z \mathrm{a}} z^{\prime}}+e^{j k_{z \mathrm{a}} z^{\prime}}\right),
$$$$
a_{22}=\frac{-1}{4 k_{z \mathrm{a}} \Gamma \sin \left(k_{z \mathrm{a}} h\right)}\left(\Gamma e^{-j k_{z \mathrm{a}} z^{\prime}}+e^{j k_{z \mathrm{a}} z^{\prime}}\right) .
$$

Coefficients above $z^{\prime}$ satisfy $a_{22}=\Gamma^{-1} a_{21}$. Since the coefficient $a_{22}$ corresponds to the wave moving away from the source, and $a_{21}$ to the wave moving towards the source, their interdependence is equivalent to the introduction of a reflection coefficient $1 / \Gamma$ at the top of the domain.

\section{Study of the instability}

\subsection{Beyond the computation domain}

The paper focuses on what happens beyond the computation domain, or how the reflection coefficient imposed at the top of the domain can be interpreted if some energy were passing through it.
One must remember that no energy should reach this limit, thanks to apodisation or absorbing layers. However, numerical uncertainties can be sufficient to imply a numerical divergence.

The question to be answered is what this upper medium should be made of to imply a reflection coefficient equal to $1 / \Gamma$. From (4), the vertical wavenumber in this medium should be

$$
k_{z \mathrm{t}}=-\epsilon k_{z \mathrm{~g}}
$$

Equivalently, the impedance into the upper domain is $Z_{\mathrm{t}}=-Z_{\mathrm{g}}$.

\subsection{Modified permittivity}

Roughness is commonly introduced by the MBV model. The reflection coefficient $\Gamma_{\bmod }$ including the roughness satisfies then

$$
\Gamma_{\bmod }=\Gamma_{0} e^{-\xi} I_{0}(\xi),
$$

where $I_{0}$ is the modified Bessel function of order 0 , and

$$
\xi \equiv \frac{1}{2}\left(\frac{4 \pi \delta_{\mathrm{r}} \sin (\theta)}{\lambda}\right)^{2},
$$

$\theta$ being the grazing angle, and $\lambda$ the wavelength. The reflection coefficient $\Gamma$ is now reformulated when the propagation medium is vacuum $\left(\varepsilon_{\mathrm{ra}}=1\right)$, with notations $k_{z \mathrm{~g}}=\beta_{\mathrm{g}}-j \alpha_{\mathrm{g}}$ and $\varepsilon_{\mathrm{rg}}=\varepsilon_{\mathrm{rg}}^{\prime}-j \varepsilon_{\mathrm{rg}}^{\prime \prime}=$ $\varepsilon_{\mathrm{rg}}^{\prime}\left(1-j \tan \delta_{\mathrm{g}}\right)$. Then,

$$
\Gamma=\frac{\left(\varepsilon_{\mathrm{rg}}^{\prime} k_{z \mathrm{a}}-\beta_{\mathrm{g}}\right)-j\left(\varepsilon_{\mathrm{rg}}^{\prime \prime} k_{z \mathrm{a}}-\alpha_{\mathrm{g}}\right)}{\left(\varepsilon_{\mathrm{rg}}^{\prime} k_{z \mathrm{a}}+\beta_{\mathrm{g}}\right)-j\left(\varepsilon_{\mathrm{rg}}^{\prime \prime} k_{z \mathrm{a}}+\alpha_{\mathrm{g}}\right)} .
$$

For taking into account the roughness into the definition of the reflection coefficient, a modified refractivity $\varepsilon_{\mathrm{rmod}}$ is introduced using (4) such that

$$
\Gamma_{\text {mod }}=\frac{\left(\varepsilon_{\mathrm{r}_{\text {mod }}^{\prime}}^{\prime} k_{z \mathrm{a}}-\beta_{\mathrm{g}}\right)-j\left(\varepsilon_{\mathrm{r}_{\text {mod }}^{\prime \prime}}^{\prime \prime} k_{z \mathrm{a}}-\alpha_{\mathrm{g}}\right)}{\left(\varepsilon_{\mathrm{r}_{\text {mod }}^{\prime}}^{\prime} k_{z \mathrm{a}}+\beta_{\mathrm{g}}\right)-j\left(\varepsilon_{\mathrm{r}_{\text {mod }}^{\prime \prime}}^{\prime \prime} k_{z \mathrm{a}}+\alpha_{\mathrm{g}}\right)},
$$

with notations

$$
\begin{aligned}
\varepsilon_{\mathrm{rmod}} & =\varepsilon_{\mathrm{rmod}}^{\prime}-j \varepsilon_{\mathrm{r} \bmod }^{\prime \prime} \\
& =\varepsilon_{\mathrm{r}_{\text {mod }}^{\prime}}^{\prime}\left(1-j \tan \delta_{\mathrm{mod}}\right) .
\end{aligned}
$$

\subsection{Limits of stability}

When $\Gamma \rightarrow 0$, the computation of the propagated field becomes unstable. For frequencies above 1 $\mathrm{GHz}, 1 / \lambda^{2}$ is much greater than 1 , and, from (8), $\Gamma_{\text {mod }}$ rapidly tends towards 0 in the spectral domain even for low $\delta_{\mathrm{r}}$.

The limit $\Gamma \rightarrow 0$ is now reformulated as

$$
\Gamma_{\mathrm{mod}} \rightarrow 0 \Leftrightarrow \tan \delta_{\mathrm{mod}} \rightarrow \frac{\alpha_{\mathrm{g}}}{\beta_{\mathrm{g}}}
$$


This late condition is also reformulated as

$$
\begin{aligned}
\tan \delta_{\text {mod }} \rightarrow \frac{\alpha_{\mathrm{g}}}{\beta_{\mathrm{g}}} & \Leftrightarrow \mathfrak{I m}\left(-\frac{k_{z \mathrm{~g}}}{\varepsilon_{\mathrm{rmod}}}\right) \rightarrow 0, \\
& \Leftrightarrow \mathfrak{I m}\left(k_{z \mathrm{t}}\right) \rightarrow 0 .
\end{aligned}
$$

This looks like the limit of a condition for finite energy at infinity:

$$
\mathfrak{I m}\left(k_{z \mathrm{t}}\right) \leq 0 .
$$

Indeed, as exposed in section 3.1 the boundary condition at the top corresponds to the introduction of a new medium above the propagating medium with impedance $-Z_{\mathrm{g}}$. And having a reflection coefficient that tends to zero on the ground is equivalent to having a non-null energy at infinity above the domain as soon as the energy reaching this interface is not null.

The following simulations highlight the relationship between unstable cases and the condition (15).

\section{Simulations}

Simulations are performed with the SSM algorithm presented in [11]. The settings are the following: computation at $3 \mathrm{GHz}$ with vertical polarisation at altitudes $z$ from 0 to $100 \mathrm{~m}$, and distances $r$ from 0 to $5 \mathrm{~km}$. The propagating medium is vacuum $\left(\varepsilon_{\mathrm{ra}}=1, \sigma_{\mathrm{a}}=0 \mathrm{~S} . \mathrm{m}^{-1}\right)$, the ground is characterised by $\varepsilon_{\mathrm{rg}}=80, \sigma_{\mathrm{g}}=0.1 \mathrm{~S} . \mathrm{m}^{-1}$. The apodisation is performed on the upper half domain, following an exponential window. Horizontal step is $100 \mathrm{~m}$. The source is Gaussian, located at $30 \mathrm{me}-$ ters high, with a $45^{\circ}$ aperture. The results shown in the following figures include the apodisation domain from $100 \mathrm{~m}$ to $200 \mathrm{~m}$. Roughness is introduced through the MBV model [8], with a height variation of the surface $\delta_{\mathrm{r}}=0.5 \mathrm{~m}$. The vertical sampling step is the varying parameter from a simulation to the other.

Three different vertical steps $\delta z$ are tested: $\delta z=$ $0.67 \mathrm{~m}, 0.40 \mathrm{~m}$, and $0.25 \mathrm{~m}$ corresponding to figures 1,2 , and 3 , respectively.

In figure 1 , the computation is stable. In figure 2 , an instability appears in the apodisation area, with a limited impact on the area of interest $(z<100$ $\mathrm{m})$. In figure 3, computation is clearly unstable. Note that the apodisation is the same in the three cases. As mentioned in section 3.3, this instability appears when the reflection coefficient $\Gamma$ tends to zero (when $\delta z$ decreases) because of the $1 / \Gamma$ term in the Green function.

In figures $4 \mathrm{a}, 4 \mathrm{~b}$, and $4 \mathrm{c},|\Gamma|$ (left ordinate) and $\tan \delta_{\mathrm{g}}-\alpha_{\mathrm{g}} / \beta_{\mathrm{g}}$ (right ordinate) are plotted with respect to $k_{z \mathrm{a}} / k_{0}$. The higher spectral components are more subject to instability. For high values of

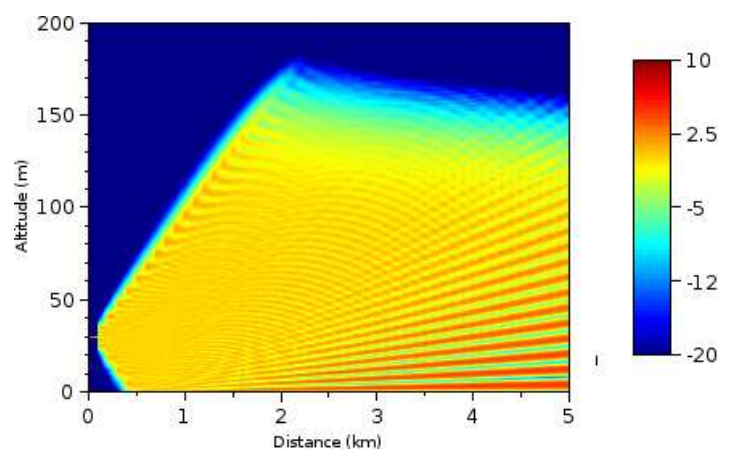

Figure 1: Propagation factor for $\delta z=0.67 \mathrm{~m}$.

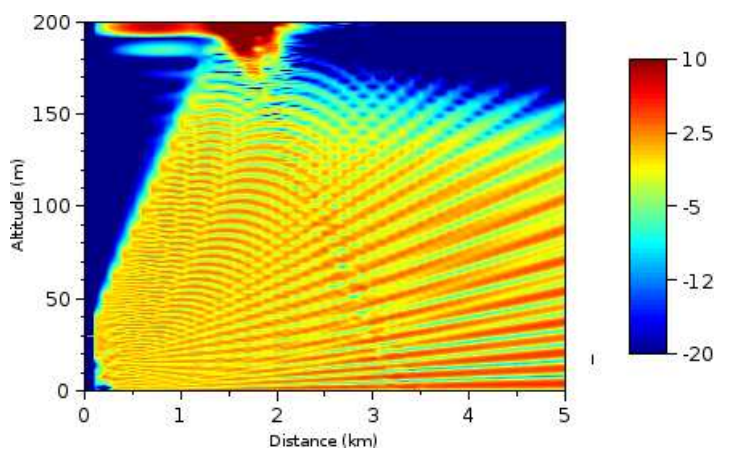

Figure 2: Propagation factor for $\delta z=0.40 \mathrm{~m}$.

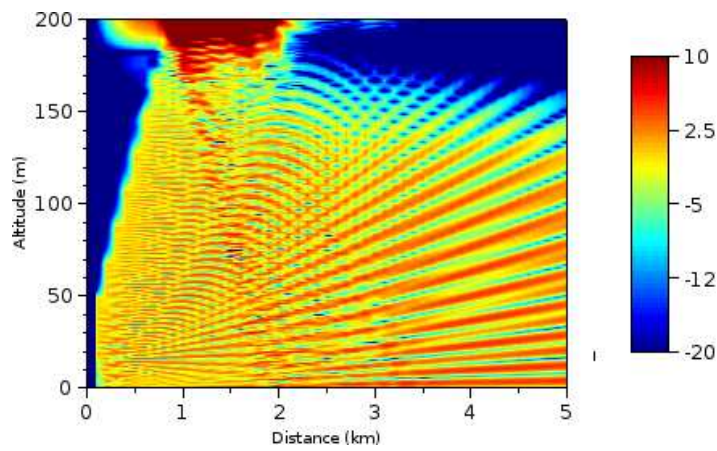

Figure 3: Propagation factor for $\delta z=0.25 \mathrm{~m}$.

$k_{z \mathrm{a}},|\Gamma|$ tends to zero (and passes to zero due to the Brewster angle), $\tan \delta_{\mathrm{g}}$ tends to $\alpha_{\mathrm{g}} / \beta_{\mathrm{g}}$ as well. As the vertical sampling step decreases, some energy is carried by spectral components hardly fulfilling the boundary condition (15), which logically leads to numerical instabilities.

\section{Conclusion}

This study shows that when the Green function is detailed for a discretised domain, the numerical divergence due to the reflection coefficient can also be seen as a radiation condition outside the computation domain that is barely fulfilled for numerous spectral components. This divergence problem is 


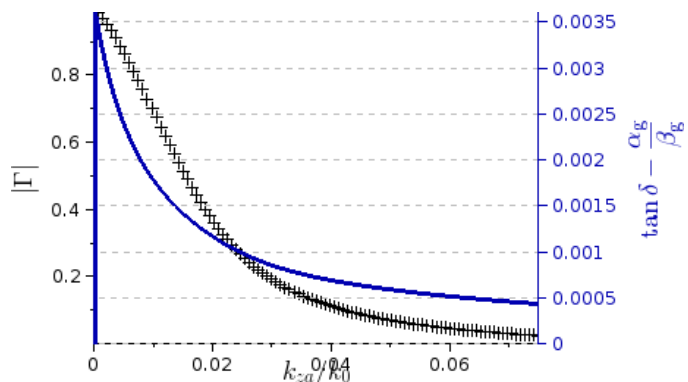

(a) $\delta z=0.67 \mathrm{~m}$

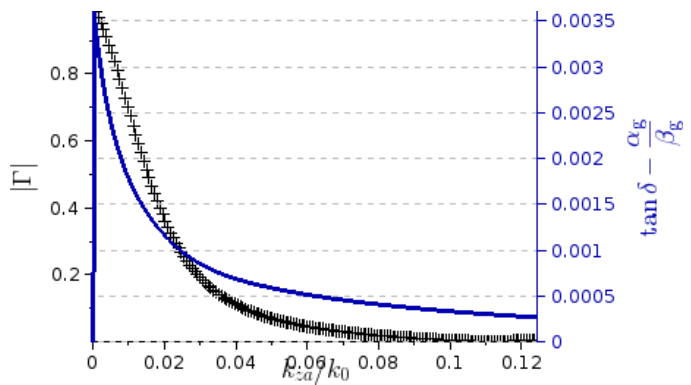

(b) $\delta z=0.40 \mathrm{~m}$

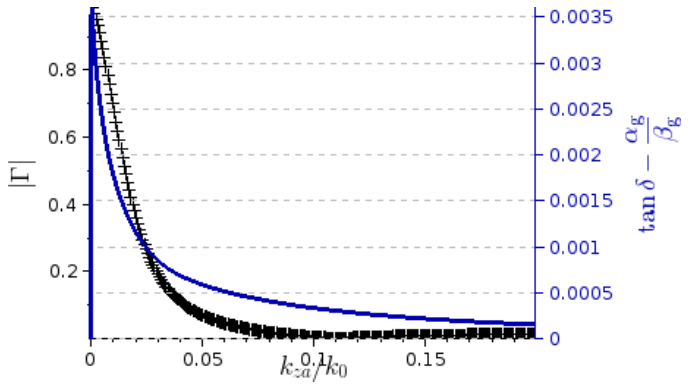

(c) $\delta z=0.25 \mathrm{~m}$

Figure 4: $|\Gamma|$ (left ordinate, cross marker, black) and $\tan \delta-\frac{\alpha_{\mathrm{g}}}{\beta_{\mathrm{g}}}$ (right ordinate, continuous line, green) with respect to $k_{z a} / k_{0}$, for three different values of $\delta z$.

still an open problem when the reflection coefficient varies with the vertical spectrum. This work should help dealing with apodisation / absorbing methods that definitely avoid numerical instabilities.

\section{References}

[1] M. Levy, Parabolic Equation Methods for Electromagnetic Wave Propagation, ser. IEE electromagnetic waves 45, IET, Ed. IEE Electromagnetic Wave Series 45, 2000.

[2] J. R. Kuttler and D. G. Dockery, "Theoretical description of the parabolic approximation/Fourier split-step method of representing electromagnetic propagation in the tropo- sphere," Radio Sci., vol. 26, no. 2, pp. 381-393, 1991.

[3] D. G. Dockery and J. R. Kuttler, "An improved impedance-boundary algorithm for Fourier split-step solutions of the parabolic wave equation," IEEE Trans. Antennas Propag., vol. 44, pp. 1592-1599, 1996.

[4] A. E. Barrios, K. Anderson, and G. Lindem, "Low altitude propagation effects a validation study of the advanced propagation model (APM) for mobile radio applications," IEEE Trans. Antennas Propag., vol. 54, pp. 28692877, 2006.

[5] G. Dockery, R. Awadallah, D. Freund, J. Gehman, and M. Newkirk, "An overview of recent advances for the TEMPER radar propagation model," in IEEE Radar Conference 2007, April 2007, pp. 896-905.

[6] J. R. Kuttler and R. Janaswamy, "Improved Fourier transform methods for solving the parabolic wave equation," Radio Sci., vol. 37, no. 2, p. 1021, 2002.

[7] J. Guo, J. Liu, K. Zhang, and Y. Long, "Improved Fourier transform parabolic equation for wave propagation over rough sea surface," in ICMMT 2008. International Conference on Microwave and Millimeter Wave Technology, 2008., vol. 2, April 2008, pp. 1025-1028.

[8] A. R. Miller, R. M. Brown, and E. Vegh, "New derivation for the rough-surface reflection coefficient and for the distribution of sea-wave elevations," in IEE Proc. H Microwaves, Optics and Antennas, vol. 131, no. 2, 1984, pp. 114-116.

[9] R. Janaswamy, "Radio wave propagation over a nonconstant immittance plane," Radio Sci., vol. 36, no. 3, pp. 387-405, 2001.

[10] A. Chabory, C. Morlaas, R. Douvenot, and B. Souny, "An exact spectral representation of the wave equation for propagation over a terrain," in ICEAA, 2012, pp. 1-4.

[11] R. Douvenot, A. Chabory, C. Morlaas, and B. Souny, "Matrix split-step resolution for propagation based on an exact spectral formulation," in ICEAA, 2012, pp. 1-4.

[12] D. G. Dudley, Mathematical Foundations for Electromagnetic Theory. IEEE Press Series on Electromagnetic Waves, 1994. 